\title{
Medición y análisis de los resultados de la prueba básica de matemática aplicada a estudiantes que ingresan a la Fundación Universidad Autónoma de Colombia ${ }^{1}$
}

\author{
CARLOS EDUARDO GÓMEZ ZÚÑIGA², RAMÓN ANTONIO OSORIO MORALES ${ }^{3}$
}

FUNDACIÓN UNIVERSIDAD AUTÓNOMA DE COLOMBIA

Recibido, octubre 15 de 2014

Concepto evaluación, noviembre 04 de 2014

Aceptado, abril 08 de 2015
Referencia: Gómez Zúñiga, C.E.; Osorio Morales, R.A. (2015). "Medición y análisis de los resultados de la prueba básica de matemática, aplicada a los estudiantes que ingresan a la Fundación Universidad Autónoma de Colombia". Revista Academia y Virtualidad, 8, (1), 66-75

\section{Resumen}

Dentro del proyecto de investigación "Indicadores de calidad en los servicios académicos ofrecidos por el Departamento de Ciencias Naturales y Exactas, basado en Control Estadístico de Procesos" se evaluaron, midieron y analizaron una serie de variables las cuales permitieron construir indicadores de calidad en los servicios académicos que ofrece dicho departamento en la Fundación Universidad Autónoma de Colombia. Una de estas variables es la "Realización curso de Matemática Introductoria”.

En este breve artículo se muestran algunos resultados generales de una prueba básica de matemáticas, aplicada a estudiantes que ingresaron a la Universidad, durante el primer semestre de 2014, la cual pretende evaluar el nivel de conocimientos matemáticos con los que llegan los estudiantes a iniciar su carrera profesional.

Los resultados obtenidos mostraron que la mayoría de estudiantes no la aprobaron. Con base en el análisis de la información obtenida se construyeron indicadores que permitirán buscar estrategias de mejoramiento del nivel académico de los estudiantes.

Palabras clave: calidad, control estadístico de procesos, indicador, prueba básica de matemáticas.

1. Artículo de investigación. Parte de los resultados del Proyecto de Investigación "Indicadores de calidad en los servicios académicos ofrecidos por el Departamento de Ciencias Naturales y Exactas, basado en control estadístico de procesos SPC".

2. Magíster en Ciencias Estadística, Universidad Nacional de Colombia, Bogotá; Licenciado en Matemáticas, Universidad de Nariño, Pasto (Colombia). Profesor Tiempo Completo, Fundación Universidad Autónoma de Colombia; investigador principal, grupo de investigación Estadística Aplicada. Contacto: cegomezu@gmail.com

3. Profesional en Administración Pública, Escuela Superior de Administración Pública, ESAP, Bogotá; Ingeniero Industrial, Fundación Universidad Autónoma de Colombia, Bogotá. Docente catedrático, Fundación Universidad Autónoma de Colombia; co-investigador grupo de investigación de Estadística Aplicada. Contacto: r.osorio_711@hotmail.com 
Carlos Eduardo Gómez Zúñiga, Ramón Antonio Osorio Morales

\section{Measurement and analysis of basic test of mathematics applied to applicants enrolled to Fundación Universidad Autónoma de Colombia}

\section{Abstract}

The research project "Quality indicators of academic services offered by Dept. of Natural and Exact Sciences based on Statistical Process Control" was used to evaluate, measure and analyze a number of variables that allowed quality indicators of academics service offered by the Dept. of Natural and Exact Sciences at Fundación Universidad Autónoma de Colombia. One of these variables is an "Introductory Mathematics Course."

This short paper shows some general results of a basic mathematics test, applied to students who entered at the University during the first half of 2014. The test is intended to assess the level of the acquired mathematical knowledge by the students to begin their career.

The results showed that most of the students have not passed the test, and based on data analysis we build indicators to look for strategies in order to improve the academic level of the students.

Keywords: basic mathematics test, indicator, quality, statistical process control.

\section{Medição e análise dos resultados da prova básica de matemática aplicada a estudantes que ingressam à Fundação Universidade Autónoma de Colômbia}

\section{Resumo}

Dentro do projeto de pesquisa "Indicadores de qualidade nos serviços académicos oferecidos pelo Departamento de Ciências Naturais e Exatas, baseado no Controle Estadístico de Processos" se avaliaram, mediram e analisaram um conjunto de variáveis, as quais permitiram a construção de indicadores de qualidade nos serviços académicos oferecidos pelo Departamento de Ciências Naturais e Exatas da Fundacional Universidade Autónoma de Colômbia. Uma dessas variáveis é a "Realização do curso de Matemática Introdutória".

Neste breve artigo mostram-se alguns resultados gerais de uma prova básica de matemática, aplicada a estudantes que ingressaram à Universidade, durante o primeiro semestre de 2014, a qual pretende avaliar o nível de conhecimentos matemáticos com que chegam os estudantes a começar a sua carreira profissional.

Os resultados obtidos mostraram que na maioria os estudantes não aprovaram-na. Baseados na análise da informação obtida construíram-se indicadores que permitirão procurar estratégias de melhoramento do nível académico dos estudantes.

Palavras-chave: qualidade, controle estadístico de processos, indicador, prova básica de matemática. 


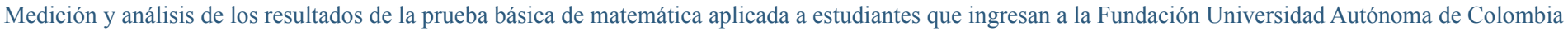

\section{Introducción}

La calidad en la educación es un tema que toma cada vez mayor relevancia en todos los niveles del sector educativo, permitiendo a través de ella crear estándares de calidad que consoliden a las instituciones de educación superior en el fortalecimiento del sistema educativo y de la acreditación tanto de programas académicos como institucional.La Fundación Universidad Autónoma de Colombia (FUAC) no es ajena a las directrices emanadas del Ministerio de Educación Nacional (MEN), razón por la cual está trabajando en el mejoramiento de la calidad de los servicios académicos ofrecidos, a través de la construcción de indicadores.

En este sentido, el presente artículo pretende mostrar parte de los resultados del proyecto de investigación denominado "Indicadores de calidad en los servicios académicos ofrecidos por el Departamento de Ciencias Naturales y Exactas basado en control estadístico de procesos SPC", mediante el cual se busca medir y evaluar la calidad de los servicios académicos ofrecidos por el Departamento de Ciencias Naturales y Exactas (DCNE), de la Fundación Universidad Autónoma de Colombia, utilizando el Control Estadístico de Procesos, que se denota como SPC, y que consiste en un conjunto de herramientas estadísticas útiles para desarrollar el mejoramiento continuo de la calidad y que van a permitir cumplir el objetivo propuesto, a través de la construcción de indicadores, que se identificarán como índices de calidad.

En el DCNE se han detectado seis variables a considerar para el desarrollo del proyecto que están directamente relacionadas con los servicios académicos ofrecidos, estas son:

- Seguimiento académico a los estudiantes.

- Caracterización del tipo de estudiante que recibe el DCNE.

- Servicio de tutorías a los estudiantes que cursan asignaturas ofrecidas por el DCNE.
- Evaluación final de los cursos mediante la aplicación de una prueba final conjunta.

- Realización curso de Matemática Introductoria.

- Realización cursos de nivelación.

La población objeto de estudio son todos los estudiantes de la FUAC que reciben los servicios prestados por el DCNE, de los cuales se seleccionó una muestra de acuerdo a cada variable. El tipo de muestreo definido es por conveniencia, en donde cada una de las variables se seleccionó en forma dirigida, realizando un análisis cuidadoso en la elección de los grupos, el tipo de datos cualitativos y/o cuantitativos que proporcionan, para que éstos tengan ciertas características acordes con los objetivos. El siguiente cuadro resume las muestras seleccionadas para cada una de las variables, la fuente y el período que se analizó.

La investigación tiene como objetivo "construir índices de calidad que permitan medir y evaluar los servicios académicos ofrecidos por el Departamento de Ciencias Naturales y Exactas (DCNE), mediante la aplicación del Control Estadístico de Procesos (SPC)", y responde a la pregunta: ¿Cómo construir índices de calidad que permitan medir y evaluar los servicios académicos ofrecidos por el Departamento de Ciencias Naturales y Exactas mediante la aplicación del Control Estadístico de Procesos (SPC)?

Para este artículo se ha decidido mostrar algunos de los resultados obtenidos en el estudio de la variable denominada: "Realización curso de Matemática Introductoria". Este curso es ofrecido a los estudiantes que ingresan a algún programa de las facultades de Ingeniería y la Facultad de Ciencias Económico Administrativas y Contables (FACEAC), y consiste en medir, en cierto grado, el nivel de conocimientos con los cuales ingresa el estudiante a la Educación Superior y relacionar los resultados con la aprobación y/o pérdida de la asignatura cursada en primer semestre.

La propuesta del proyecto consiste en que los indicadores que de allí surjan, se apliquen y pongan en práctica al 


\begin{tabular}{|c|c|c|c|}
\hline Variables & Muestra & Fuente & Período \\
\hline 1. Seguimiento académico a los estudiantes. & $\begin{array}{l}\text { Una clase por cada una de las asignaturas que ofrece } \\
\text { el DCNE en ambas jornadas diurna y nocturna. }\end{array}$ & Secundaria & $2013-2$ \\
\hline $\begin{array}{l}\text { 2. Caracterización del tipo de estudiante } \\
\text { que recibe el DCNE. }\end{array}$ & $\begin{array}{l}\text { Todas las clases de Calculo Diferencial en jornada } \\
\text { diurna y nocturna, que corresponden a la Facultad } \\
\text { de Ingeniería. }\end{array}$ & Primaria & $2013-2$ \\
\hline $\begin{array}{l}\text { 3. Servicio de tutorías a los estudiantes } \\
\text { que cursan asignaturas ofrecidas por el } \\
\text { DCNE. }\end{array}$ & $\begin{array}{l}\text { Total de tutorías realizadas por los docentes de } \\
\text { Tiempo Completo (6). }\end{array}$ & Primaria & $2013-1$ y $2013-2$ \\
\hline $\begin{array}{l}\text { 4. Evaluación final de los cursos mediante } \\
\text { la aplicación de una prueba final con- } \\
\text { junta. }\end{array}$ & $\begin{array}{l}\text { Una clase por cada una de las asignaturas que ofrece } \\
\text { el DCNE en ambas jornadas diurna y nocturna. }\end{array}$ & Primaria & $2014-1$ \\
\hline $\begin{array}{l}\text { 5. Realización curso de Matemática Intro- } \\
\text { ductoria. }\end{array}$ & La totalidad de cursos de matemática introductoria. & Primaria & $2014-1$. \\
\hline 6. Realización cursos de nivelación. & $\begin{array}{l}\text { Los cursos de nivelación realizados en los períodos } \\
\text { intersemestrales junio-julio. }\end{array}$ & Primaria & Años 2012 y 2013. \\
\hline
\end{tabular}

Tabla 1. Información de variables seleccionadas.

Fuente: Información recolectada a través del desarrollo del proyecto.

interior del DCNE. Finalmente, a través de su ejecución, verificación y posteriores correctivos, se espera que sean aplicados al interior de la FUAC.

\section{Conceptos básicos}

\section{Control Estadístico de Procesos (SPC)}

"El SPC, es un conjunto poderoso de herramientas para resolver problemas de un producto o servicio durante el proceso de fabricación o ejecución, muy útil para conseguir la estabilidad y mejorar la capacidad de un proceso ya sea de manufactura, o fuera de ella, mediante la reducción de la variabilidad". (Montgomery, D., 2010).

El SPC puede aplicarse a cualquier proceso y dentro de él se reconocen siete herramientas principales, mediante las cuales se puede obtener información que permita evaluar el desempeño de los servicios ofrecidos por el DCNE y a su vez, generar indicadores que contextualizados al ámbito educativo, llevan al concepto de indicador de gestión.
Estas herramientas son:

1. Histograma o diagrama de tallo y hoja.

2. Hoja de verificación.

3. Gráfica de Pareto.

4. Diagrama de causa y efecto.

5. Diagrama de concentración de defectos.

6. Diagrama de dispersión.

7. Cartas de control.

Para la variable de interés, Realización curso de Matemática Introductoria se utilizaron la Hoja de verificación y el Histograma.

La información de la variable es obtenida a través de los cursos de matemática introductoria ofrecidos durante el primer semestre de 2014 y se considera como fuente de información primaria. Para entender el alcance del SPC, se debe tener claridad sobre lo que significa Proceso, Control, Estadística, Calidad e Indicador, conceptos que se definen de manera puntual a continuación: 


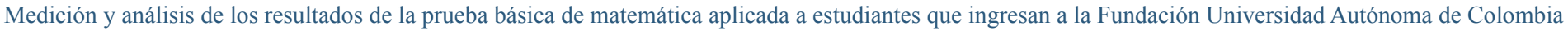

\section{Proceso}

Es el conjunto de actividades en un orden lógico que persigue un objetivo común, transformando entradas en salidas, con el apoyo de recursos. El término Proceso se considera como cualquier servicio administrativo o académico que corresponde a la secuencia: Entrada - Actividad - Salida. (Bissell, 1994, citada por Vargas, 2006) describe un proceso como la actividad de producir entradas en salidas (inputs into outputs).

\section{Control}

Según Bissell, la idea de control no es simplemente la de monitoreo; en este sentido se puede ampliar el concepto de control como un ciclo PHVA (Planear, Hacer, Verificar y Actuar), a través del cual el control es ejercido en cada una de sus etapas para terminar con éxito un proceso dentro de la gestión académica y/o administrativa.

\section{Estadística}

Es la ciencia de analizar datos y sacar conclusiones, tomando en consideración la variación de los mismos. El término Estadístico se considera como todas aquellas técnicas o herramientas estadísticas que se necesitan para determinar si en un proceso o en un servicio, ha ocurrido alguna variación anormal y buscar reducir la variabilidad de éstos, para detectar cambios en los parámetros del proceso e identificar factores que están afectando las características del mismo. (Montgomery D., 2010).

\section{Calidad}

Aunque este concepto y la demanda por productos y servicios de calidad han sido parte de la naturaleza humana por mucho tiempo, la cuantificación de la calidad y el establecimiento de estándares de calidad es un fenómeno del siglo XX. Varias definiciones de calidad se encuentran en la literatura: W. Edwards Deming expresa que la calidad se debe definir en términos de satisfacción del consumidor. Plantea además que la calidad de un producto o servicio se debe establecer en forma multidimensional. Explicó la calidad, definiéndola como una red de componentes interdependientes que trabajan juntas, con el propósito de lograr los objetivos propuestos por el sistema. Para Joseph M. Juran, la calidad consiste en aquellas características del producto o servicio que satisfacen las necesidades del consumidor. Insiste además en que hablar de calidad equivale a hablar de un producto o servicio sin defectos. Kaoru Ishikawa concuerda también en la definición de calidad como satisfacción del consumidor, pero insiste en que el concepto de calidad debe abarcar todos los eslabones de la organización (Vargas, 2010).

\section{Indicador}

Un indicador se puede definir como: "Un elemento informativo de carácter cuantitativo, sobre algún componente o atributo de una realidad, orientado a servir de fundamento para elaborar juicios sobre ella" (Pérez, J., 2002, p. 62).

\section{Medición y análisis de los resultados de la prueba básica de matemática, aplicada a los estudiantes que ingresan a la FUAC}

Realización curso de Matemática Introductoria: Este curso es realizado por el DCNE desde hace varios años, y consiste en recordar y afianzar los conceptos básicos matemáticos, aprendidos durante la Educación Básica. Para esta variable se tomó la totalidad de cursos de matemática introductoria, correspondiente al primer período académico de 2014.

Para obtener la información se diseñó y aplicó una prueba de conocimientos básicos, a todos los estudiantes que ingresaron a la Universidad en este período y que realizaron o no el curso. Con esta información se crearon indicadores que permitieron, entre otros, determinar el nivel de conocimientos con los cuales ingresan los estudiantes de las Facultades de Ingeniería y FACEAC; con los resultados obtenidos se busca proponer mecanismos que nivelen a los estudiantes en sus competencias matemáticas.

A continuación se muestra la hoja de verificación y la figura de los resultados generales de la prueba: 
Carlos Eduardo Gómez Zúñiga, Ramón Antonio Osorio Morales

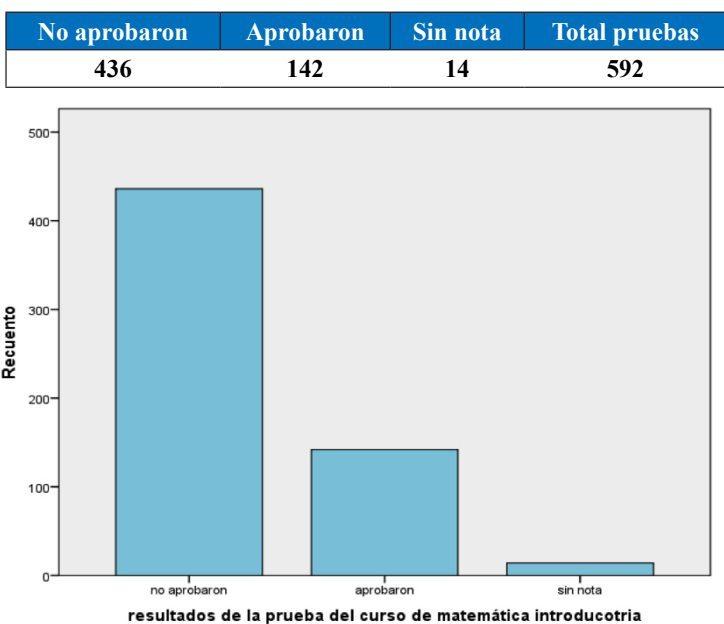

Figura 1. Resultados de la prueba usando SPSS.

Fuente: Prueba de Matemática Introductoria aplicada a estudiantes nuevos, en el desarrollo del proyecto.

Lo anterior muestra que un gran número de estudiantes no aprueba la evaluación de conocimientos básicos.

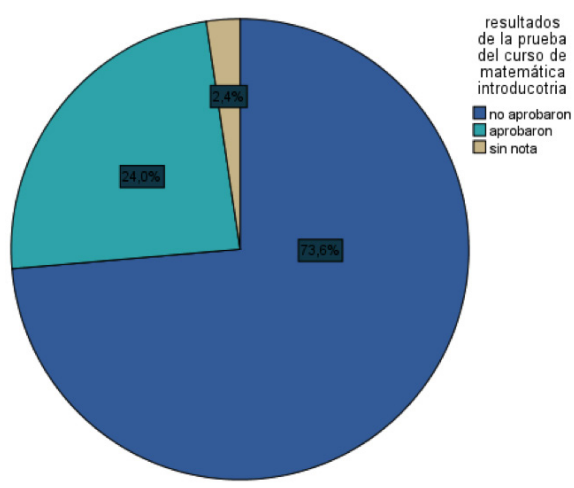

Figura 2. Porcentaje de aprobación usando SPSS.

Fuente: Resultados de la Prueba de Matemática Introductoria aplicada a estudiantes nuevos, en el desarrollo del proyecto.

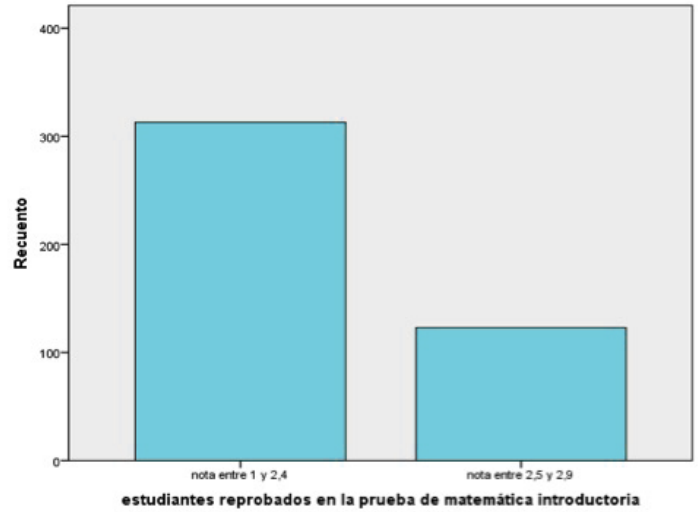

Figura 3. Estudiantes reprobados usando SPSS

Fuente: Resultados de la Prueba de Matemática Introductoria aplicada a estudiantes nuevos, en el desarrollo del proyecto.
Aquí se observa que del total de estudiantes reprobados, 313 correspondiente al 72\%, obtuvieron una calificación menor a 2.4, lo que indica un bajo rendimiento en la prueba. Una consecuencia de este resultado implica bajo rendimiento en el área de matemáticas en el inicio del semestre académico. Para disminuir la deserción se deben planear estrategias de mejoramiento, tales como tutorías obligatorias.

Ahora, de la siguiente hoja de verificación y su correspondiente figura se observa la calificación promedio y el número de estudiantes por programa:

\begin{tabular}{|c|c|c|}
\hline PROGRAMA & $\begin{array}{c}\text { NÚMERO DE } \\
\text { ESTUDIANTES }\end{array}$ & $\begin{array}{c}\text { CALIFICACIÓN } \\
\text { PROMEDIO }\end{array}$ \\
\hline Administración & 98 & 2,1 \\
Ambiental & 96 & 2,7 \\
\hline Contaduría & $\mathbf{8 9}$ & $\mathbf{2 , 3}$ \\
\hline Economía & 37 & $\mathbf{2 , 2}$ \\
\hline Electromecánica & $\mathbf{3 1}$ & $\mathbf{2 , 8}$ \\
\hline Electrónica & $\mathbf{1 8}$ & $\mathbf{3}$ \\
\hline Industrial & $\mathbf{1 0 8}$ & $\mathbf{2 , 7}$ \\
\hline REI & 91 & $\mathbf{2 , 3}$ \\
\hline Sistemas & 19 & $\mathbf{2 , 6}$ \\
\hline En blanco & $\mathbf{5}$ & \\
\hline
\end{tabular}

Fuente: Resultados de la Prueba de Matemática Introductoria aplicada a estudiantes nuevos, en el desarrollo del proyecto.

\section{Promedio de nota por carrera}

口Estudiantes $\quad$ Calificación Promedio

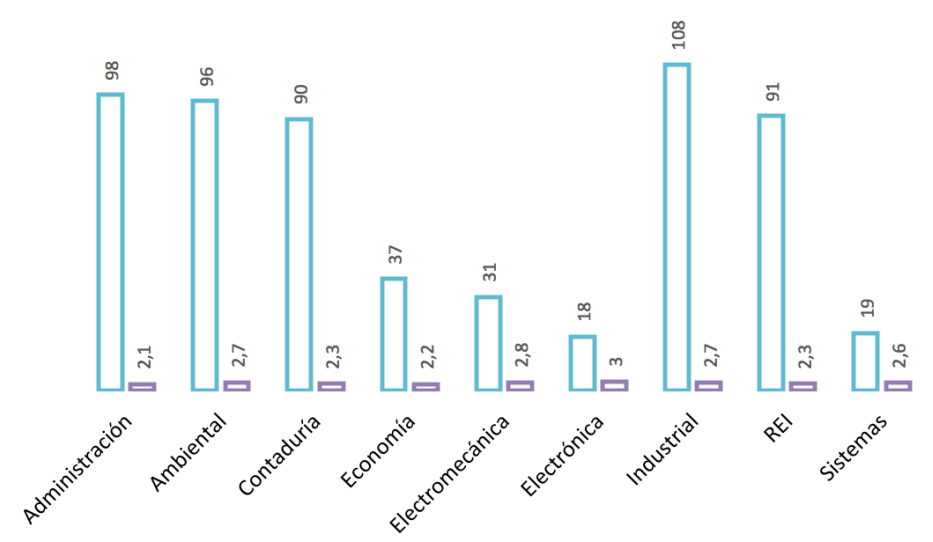

Figura 4. Promedio de notas por carrera. Usando Excel.

Fuente: Resultados de la Prueba de Matemática Introductoria aplicada a estudiantes nuevos, en el desarrollo del proyecto. 
Aquí se observa que la calificación promedio de los 18 estudiantes de Ingeniería Electrónica, fue de 3.0, siendo el único programa, que aprobó la prueba de matemáticas básicas.Además se observa que los 98 estudiantes de Administración de Empresas, fueron los que obtuvieron la menor nota promedio, con una calificación de 2.1. Con base en los resultados obtenidos, se procedió a construir los siguientes indicadores:

\section{Variable: Realización curso de Matemática Introductoria \\ HOJA DE VIDA INDICADOR No. 1 - ESTUDIANTES QUE REPRUEBAN \\ LA PRUEBA BÁSICA DE MATEMÁTICAS}

\begin{tabular}{|c|c|}
\hline NOMBRE: & $\begin{array}{l}\text { ESTUDIANTES QUE REPRUEBAN LA PRUEBA BÁSICA DE } \\
\text { MATEMÁTICAS }\end{array}$ \\
\hline OBJETIVO: & $\begin{array}{l}\text { Medir el nivel de conocimientos matemáticos básicos con los cuales llegan los } \\
\text { estudiantes a la Educación Superior. }\end{array}$ \\
\hline DESCRIPCIÓN: & $\begin{array}{l}\text { Se realiza una prueba de conocimientos básicos en matemáticas que incluyen } \\
\text { preguntas de aritmética, álgebra, geometría y lectura estadística. }\end{array}$ \\
\hline FUENTE DE DATOS: & $\begin{array}{l}\text { Prueba básica de conocimientos matemáticos aplicados a los estudiantes que } \\
\text { ingresan a cursar primer semestre en uno de los programas de las facultades de } \\
\text { Ingeniería y FACEAC. }\end{array}$ \\
\hline CALCULO: & $\begin{array}{l}\text { Número de estudiantes con nota inferior a } 3.0 \text {, dividido entre el total de } \\
\text { estudiantes que presentan la PBM. }\end{array}$ \\
\hline UNIDAD: & Porcentaje. \\
\hline PERIODICIDAD: & Semestral. \\
\hline RESPONSABLE: & DCNE. \\
\hline META: & $\begin{array}{l}\text { Aplicar la PBM al inicio de cada semestre académico, con el fin de determinar } \\
\text { el nivel de conocimientos en matemáticas con los cuales ingresan los estudiantes } \\
\text { a la Universidad y buscar estrategias que mejoren su rendimiento académico. }\end{array}$ \\
\hline OBSERVACIONES: & \\
\hline
\end{tabular}




\section{Variable: Realización curso de Matemática Introductoria}

HOJA DE VIDA INDICADOR No. 2 - PROMEDIO DE NOTA POR

\section{PROGRAMA EN LA PRUEBA BÁSICA DE MATEMÁTICAS}

\begin{tabular}{|c|c|}
\hline NOMBRE: & $\begin{array}{l}\text { PROMEDIO DE NOTA POR PROGRAMA EN } \\
\text { LA PRUEBA BÁSICA DE MATEMÁTICAS }\end{array}$ \\
\hline OBJETIVO: & $\begin{array}{l}\text { Medir el rendimiento académico en las pruebas de matemáticas básicas aplicadas } \\
\text { a los estudiantes que ingresan a la FUAC, calculando las calificaciones promedio } \\
\text { obtenidas en la PBM, de acuerdo al programa en el cual se matriculan. }\end{array}$ \\
\hline DESCRIPCIÓN: & $\begin{array}{l}\text { Se realiza una prueba de conocimientos básicos en matemáticas que incluyen } \\
\text { preguntas de aritmética, álgebra, geometría y lectura estadística. }\end{array}$ \\
\hline FUENTE DE DATOS: & $\begin{array}{l}\text { Prueba básica de conocimientos matemáticos aplicados a los estudiantes que } \\
\text { ingresan a cursar primer semestre en uno de los programas de las facultades de } \\
\text { Ingeniería y FACEAC. }\end{array}$ \\
\hline CALCULO: & $\begin{array}{l}\text { Suma de calificaciones por programa, dividido entre el total de estudiantes que } \\
\text { presentan la PBM. }\end{array}$ \\
\hline UNIDAD: & Calificación Promedio. \\
\hline PERIODICIDAD: & Semestral. \\
\hline RESPONSABLE: & DCNE. \\
\hline META: & $\begin{array}{l}\text { Aplicar la PBM al inicio de cada semestre académico, con el fin de determinar } \\
\text { el nivel de conocimientos en matemáticas con los cuales ingresan los estudiantes } \\
\text { a la Universidad y buscar estrategias por programa o facultad, que mejoren su } \\
\text { rendimiento académico. }\end{array}$ \\
\hline OBSERVACIONES: & \\
\hline
\end{tabular}

\section{Conclusiones}

Los resultados que muestra el análisis estadístico de los datos no son otra cosa que la tendencia generalizada que registra la educación superior en Colombia, consecuencia de una deficiente calidad en la educación básica y media. Esto permite concluir que el nivel de conocimientos matemáticos con los cuales llegan los estudiantes a la
Universidad es bajo. Además, varias características pudieron ser determinadas en una encuesta que se aplicó en el mismo momento de la realización de la PBM. En ella se pudo establecer, entre otras:

- El tiempo transcurrido entre la finalización de los estudios de la educación básica y media, y el ingreso a la educación superior, como mostró el estudio que 


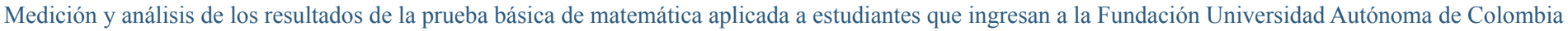

un 37.7\% de estudiantes que ingresaron en el 2014, cursaron estudios entre e1979 y 2010.

- Estudiantes que fueron cobijados con la Ley 30 (Promoción automática), correspondientes a los años de 2000 a 2011, con un porcentaje del $47.43 \%$.

- Bajo nivel matemático recibido durante la educación básica y media, lo que se evidencia que un $23.36 \%$ de estudiantes reprobraron la prueba de Matemática Introductoria.

- Falta de definición en la elección de programa a estudiar.

Existen otros factores que pueden afectar el nivel de conocimientos con los cuales los estudiantes asumen el compromiso de la educación superior, como son: situación laboral, lugar de origen, fuente de sustento, estado civil, personas a cargo, falta de orientación profesional, tipo de establecimiento de procedencia, entre otros, factores que la FUAC deberá tener en cuenta al momento de la inscripción y aceptación de los mismos en cualquiera de los programas que ésta ofrece.

El reto para las Instituciones de Educación Superior (IES) que reciben estudiantes con estas características, es generar estrategias que permitan no sólo aumentar el nivel académico de los mismos, sino también retenerlos durante toda su carrera, para que al final de ella sean profesionales exitosos y competitivos en el medio laboral.

Como conclusión en lo referente al uso de la estadística para realizar este tipo de investigaciones, se puede decir que es una herramienta útil mediante la cual se obtiene un conjunto o patrones de información, que a través de un proceso busca modelar situaciones o parámetros que se constituyen en información válida, para ser utilizada posteriormente en la implementación de los indicadores de calidad.

Dado que la información obtenida de las fuentes de datos primarias y secundarias es bastante amplia, se ve la necesidad del uso de software estadístico, para el caso de la presente investigación, el SPSS, software que brinda muchas herramientas y que permitirá tomar decisiones al momento de la construcción de los indicadores de calidad.

\section{Referencias}

Álvarez, J. (1997). Etiología de un sueño o el abandono de la universidad por parte de los estudiantes por factores no académicos. Bogotá: Universidad Autónoma de Colombia.

Artunduaga, M. (2008). Variables que influyen en el rendimiento académico en la universidad. Universidad Complutense de Madrid. Departamento de Métodos de Investigación y Diagnóstico en Educación.

Bissell, D. (1994). Statistical Methods for SPC and TQM. Londres: Chapman and Hall.

Cano, E. (1998). Evaluación de la Calidad Educativa. Madrid: La Muralla.

Centro de Estudios Internacionales para el Desarrollo, CEID. (2009).

Cominetti, R. y Ruiz, G. (1997). Algunos Factores del Rendimiento: Las expectativas y el género. En: Revista Iberoamericana de Educación Human Development. Department. LCSHD. Paper. Series, 20. Mayo. México: Universidad Cristóbal Colón.

Departamento de Bienestar Universitario, Área de Promoción socioeconómica. Notas Universitarias No. 2. (2010). Deserción estudiantil: El caso de la Universidad Autónoma de Colombia. Bogotá: Universidad Autónoma de Colombia.

Departamento de Ciencias Naturales y Exactas. (2004). Memorias Trabajo Realizado Durante el Período Intersemestral. Universidad Autónoma de Colombia. Bogotá.

Departamento de Ciencias Naturales y Exactas, DCNE. (2014). Fundación Universidad Autónoma de Colombia. Bogotá. 
Duncan, A. (1989). Control de calidad y estadística industrial. México: Alfaomega.

Ministerio de Educación Nacional (MEN). (2013). Estructura de Indicadores de Perfilación y Caracterización para las Instituciones de Educación Superior (IES). Bogotá: MEN.

Montgomery, D. (2010). Control estadístico de la Calidad. México: Limusa Wiley.

Vargas, A. (2006). Control estadístico de calidad. Bogotá: Universidad Nacional de Colombia, Unibiblos.

Vargas, A., Yañez, S. \& González, N. (2011). Cartas de Control $\mathrm{T}^{2}$ multivariadas usando $\mathrm{R}$ y SAS. Bogotá: Universidad Nacional de Colombia, Unibiblos 